\title{
BIBLIOGRAPHY
}

1. M. Arkowitz and C. Curjel, The group of homotopy equivalences of a space, Bull. Amer. Math. Soc. 70 (1964), 293-296.

2. Sze-tsen Hu, Homotopy theory, Academic Press, New York, 1959.

3. L. K. Hua and I. Reiner, On the generators of the symplectic modular group, Trans. Amer. Math. Soc. 65 (1949), 415-426.

4. P. J. Kahn, Characteristic numbers and oriented homotopy type, Topology 3 (1965), 81-95. 127.

5. P. Olum, Self-equivalences of pseudo-projective planes, Topology 4 (1965), 109-

6. D. Puppe, Homotopiemengen und ihre induzierten Abbildungen. I, Math. Z. 69 (1958), 299-344.

7. Weishu Shih, On the group $\mathcal{E}[\mathrm{X}]$ on homotopy equivalence maps, Bull. Amer. Math. Soc. 70 (1964), 361-365.

8. C. T. C. Wall, Classification of (n-1)-connected 2n-manifolds, Ann. of Math. (2) 75 (1962), 163-182.

CORNELl UNIVERSity

\section{THE SOLUTION BY ITERATION OF LINEAR FUNCTIONAL EQUATIONS IN BANACH SPACES ${ }^{1}$}

BY F. E. BROWDER AND W. V. PETRYSHYN

Communicated December 20, 1965

Let $X$ be a Banach space (real or complex), $T$ a bounded linear operator from $X$ to $X$. We are concerned with the solution of the equation

$$
u-T u=f,
$$

by the iteration process of Picard-Poincare-Neumann,

$$
x_{n+1}=T x_{n}+f \quad\left(x_{0} \text { given }\right),
$$

i.e. with the convergence of the sequence

$$
x_{n}=T^{n} x_{0}+\left(f+T f+\cdots+T^{n-1} f\right) .
$$

By an earlier result of the first-named author (Browder [2]), if $X$ is reflexive, a solution $u$ for the equation (1) will exist for a given element $f$ of $X$ and an operator $T$ which is asymptotically bounded (i.e. $\left\|T^{k}\right\| \leqq M$ for some $M>0$ and all $k \geqq 1$ ) if and only if the sequence $\left\{x_{n}\right\}$ is bounded for any fixed $x_{0}$. Our object in the present paper is to

1 The preparation of this paper was partially supported by NSF Grant GP-3552. 
sharpen this conclusion under a slightly sharper hypothesis on $T$ (without any assumption of reflexivity on $X$ ) and to derive a number of interesting applications of this result in the domain of numerical functional analysis.

THEOREM 1. Let $X$ be a Banach space, $T$ a bounded linear operator in $X$ which is asymptotically convergent, i.e. $T^{k} x$ converges in $X$ as $k \rightarrow \infty$ for each $x$ in $X$. Then:

(a) If $f$ is an element of the range of $(I-T)$, the sequence $\left\{x_{n}\right\}$ defined above for any initial approximation $x_{0}$ will converge to a solution $u$ of the equation $u-T u=f$.

(b) If any subsequence $\left\{x_{n_{j}}\right\}$ of the sequence converges to an element $y$ of $X$, then $y$ is a solution of $y-T y=f$, and the whole sequence converges.

(c) If $X$ is reflexive and the sequence $\left\{x_{n}\right\}$ is bounded, then the sequence $\left\{x_{n}\right\}$ converges to a solution of the equation (1).

REMARK. Let us underline the practical importance of the conclusion (b) of Theorem 1, which allows the testing of the convergence of the Picard iteration sequence by any infinite subsequence, a method which is not obviously applicable without Theorem 1.

Proof of Theorem 1. Since $T^{n} x \rightarrow Q x$ for each $x$ in $X$, it follows from the uniform boundedness theorem that there exists $M$ such that $\left\|T^{n}\right\| \leqq M$ for all $n \geqq 1$, while $Q$ is a bounded linear operator from $X$ to $X$. For each $w$ in the conjugate space $X^{*}$ of $X$, we have

$$
\left(x,\left(T^{*}\right)^{n} w\right)=\left(T^{n} x, w\right) \rightarrow(Q x, w)=\left(x, Q^{*} w\right),
$$

i.e. $\left(T^{*}\right)^{n} w$ converges weakly to $Q^{*} w$. Hence

$$
\left(T^{2 n} x, w\right)=\left(T^{n} x,\left(T^{*}\right)^{n} w\right) \rightarrow\left(Q x, Q^{*} w\right)=\left(Q^{2} x, w\right),
$$

from which it follows that $T^{2 n} x$ converges weakly to $Q^{2} x$, i.e. $Q=Q^{2}$. Moreover $Q T x=\lim _{n} T^{n+1} x=Q x=T Q x$, i.e. $Q=Q T=T Q$. Thus, $Q$ is an idempotent whose range is contained in $N(I-T)$, the nullspace of $I-T$, and obviously coincides with this nullspace.

Proof OF (a). Suppose $f$ lies in $R(I-T)$, the range of $(I-T)$. Then $f=u-T u$ for some $u$ in $X$. Let

$$
S_{n}(f)=\sum_{j=0}^{n-1} T^{j}(f) .
$$

We have

$$
x_{n}=T^{n} x_{0}+S_{n}(f)
$$


and

$$
S_{n}(f)=u-T^{n} u .
$$

Since $T^{n} x_{0} \rightarrow Q x_{0}, T^{n} u \rightarrow Q u$, it follows that $x_{n} \rightarrow u-Q\left(u-x_{0}\right)$, which is a solution of equation (1).

Proof OF (b). Suppose $\left\{x_{n_{j}}\right\}$ is a convergent subsequence of $x_{n}$ with limit $y$. Then:

$$
x_{n_{j}}=T^{n_{j}} x_{0}+S_{n_{j}}(f)
$$

and $S_{n_{j}}(f) \rightarrow y-Q x_{0}$. Since

$$
Q x_{n_{j}}=Q T^{n_{i}} x_{0}+Q S_{n_{j}}(f)=Q x_{0}+n_{j} Q f,
$$

it follows that $Q f=0$. Moreover, we have

$$
(I-T) S_{n_{j}}(f)=f-T^{n_{j}} f \rightarrow f-Q f=f .
$$

Hence

$$
(I-T)\left(y-Q x_{0}\right)=(I-T) y=f .
$$

q.e.d.

Proof OF (c). By the result of [2], if $X$ is reflexive and $T$ is asymptotically bounded, the boundedness of $\left\{x_{n}\right\}$ implies that $f$ lies in $R(I-T)$. We then apply (a). q.e.d.

We pass now to the verification of the hypotheses of Theorem 1 for particular classes of operators $T$. A useful auxiliary result for this purpose is the following:

Theorem 2. Let $T$ be a continuous linear operator on a Banach space $X$ for which $(-1)$ is not an eigenvalue and such that $T^{2 n} x$ converges as $n \rightarrow \infty$ for each $x$ in $X$. Then $T$ is asymptotically convergent and satisfies the conclusions of Theorem 1 :

Proof of Theorem 2. By hypothesis, $T^{2 n} x$ converges to $Q x$. Furthermore, $T^{2 n+1} x=T^{2 n}(T x)$ converges to $Q(T x)$. It follows as in the proof of Theorem 1 that $Q$ is a projection operator on $N\left(I-T^{2}\right)$ and that $Q$ commutes with $T$. Since $(-1)$ is not an eigenvalue of $T, N\left(I-T^{2}\right)=N(I-T)$. Thus $Q T=Q$, and $T^{n} x \rightarrow Q x$. q.e.d.

REMARK. For $T$ to be asymptotically convergent, it is necessary that $(-1)$ not be an eigenvalue of $T$.

THEOREM 3. Let $H$ be a Hilbert space, $T$ a self-adjoint contraction on $H$, i.e. $\|T\| \leqq 1$. Then $T$ is asymptotically convergent if and only if $(-1)$ is not an eigenvalue of $T$. More generally, the same conclusion holds if $T$ is a scalar operator in the sense of Dunford [3] which has real 
spectrum and is asymptotically bounded. In particular, the results of Theorem 1 hold for these classes of operators.

Proof of Theorem 3. If $T$ is self-adjoint with $\|T\| \leqq 1$, then $0 \leqq T^{2} \leqq I$. Thus $T^{2 n}$ is a monotone decreasing sequence of nonnegative self-adjoint operators and therefore strongly convergent $[6, p$. 261]. We apply Theorem 2 to complete the self-adjoint case.

If $T$ is a scalar operator in the sense of Dunford with real spectrum on a Hilbert space $H, T=A S A^{-1}$ for a self-adjoint operator $S$ and $A$ continuously invertible. Then $T^{n}=A S^{n} A^{-1}$, and it suffices to show that $\|S\| \leqq 1$, since $S$ has the same spectrum as $T$, and the same eigenvalues. Since $T$ is asymptotically bounded, so is $S$, but a self-adjoint operator $S$ is asymptotically bounded if and only if $\|S\| \leqq 1$. q.e.d.

Theorem 3 for the self-adjoint case is a strengthening of results of Krasnoselski [4], Bialy [1], and Petryshyn [5]. Unlike the proofs of Krasnoselski and Bialy, in particular, no detailed use is made of the explicit spectral representation of $T$. The passage from convergence of a subsequence to convergence of the whole sequence seems new even in the cases already treated in the above papers.

We now give another application outside of the Hilbert space framework, and based on results of E. M. Stein [8] and G. C. Rota [7].

Theorem 4. Let $(\Omega, \Sigma, \mu)$ be a positive measure space, $T$ a selfadjoint operator in $L^{2}(\mu)$ which maps $L^{1}(\mu)$ into itself with norm $\leqq 1$. Then for any $p$ with $1<p<\infty$, if $(-1)$ is not an eigenvalue of $T$ in $L^{p}(\mu)$, then $T$ is asymptotically convergent in $L^{p}(\mu)$ and the conclusions on the convergence of the Picard approximants given in Theorem 1 are valid for $T$ in $L^{p}(\mu)$.

Proof of Theorem. By Theorem 2, it suffices to show the convergence of $S^{n}$ with $S=T^{2}$. $S$ is a nonnegative self-adjoint operator in $L^{2}(\mu)$ and its norm in $L^{1}(\mu)$ is not greater than 1 . Hence by Corollary 1 , p. 1895 of [8], $S^{n}$ converges strongly in $L^{p}(\mu)$ for every $p$ with $1<p<\infty$. q.e.d.

As a final application, we give two results of interest in numerical applications.

TheOREM 5. Let $T$ be a self-adjoint contraction in a Hilbert space $H$ with (-1) not an eigenvalue of $T$. Consider the iteration scheme for any $x_{0}$ in $H$ given by

$$
y_{k}=T^{2^{k-1}} y_{k-1}+U_{2^{k-1}}(f), \quad y_{0}=x_{0},
$$


with

$$
U_{2^{k-1}}=\left(I+T^{2^{k-2}}\right) U_{2^{k-2}} \quad U_{1}=I .
$$

Then $\left\{y_{k}\right\}$ converges if and only if the equation $u-T u=f$ has $a$ solution.

Proof of Theorem 5. The element $y_{k}=x_{2} k$ for the Picard sequence.

TheoRem 6. Let $H$ be a Hilbert space, $B$ a bounded linear operator in $H, \alpha$ a real parameter such that $0<\alpha<2 /\left\|B^{*} B\right\|$, and let $P$ denote the orthogonal projection of $H$ onto the closure of $R(B)$. For any given $x_{0}$ in $H$, determine the sequence $\left\{u_{k}\right\}$ by the process

$$
u_{0}=x_{0}, \quad u_{k}=T^{2^{k-1}} u_{k-1}+U_{2^{k-1}} f,
$$

where

$$
f=-\alpha B^{*} g, \quad T=I-\alpha B^{*} B,
$$

and $U_{2^{k}}$ is defined in terms of $T$ as in Theorem 5. Then $\left\{u_{k}\right\}$ converges to a solution of $B x=P g$ if and only if the latter equation has a solution.

The proof of Theorem 6 is obtained by applying Theorem 5 to the self-adjoint operator $\mathrm{T}$.

\section{BIBLIOGRAPHY}

1. H. Bialy, Iterative Behandlung linearer Funktionalleichungen, Arch. Rational Mech. Anal. 4 (1959), 166-176.

2. F. E. Browder, On the iteration of transformations in noncompact minimal dynamical systems, Proc. Amer. Math. Soc. 9 (1958), 773-780.

3. N. Dunford, Spectral operators, Pacific J. Math. 4 (1954), 321-354.

4. M. A. Krasnoselskiy, On the solution of equations with self-adjoint operators by the method of successive approximations, Uspehi Mat. Nauk 15 (1960), 161-165.

5. W. V. Petryshyn, The generalized overrelaxation method for the approximate solution of operator equations in Hilbert space, J. Soc. Indust. Appl. Math. 10 (1962), 675-690.

6. F. Riesz and B. Sz-Nagy, Lecons d'analyse fonctionnelle, Akadémiai Kiado, Budapest, 1952.

7. G. C. Rota, An "altermierende Verfahren" for general positive operators, Bull. Amer. Math. Soc. 68 (1962), 95-102.

8. E. M. Stein, On the maximal ergodic theorem, Proc. Nat. Acad. Sci. U.S.A. 47 (1961), 1894-1897.

University of Chicago 